NOTICE: this is the author's version of a work that was accepted for publication in Electrochimica Acta. Changes resulting from the publishing process, such as peer review, editing, corrections, structural formatting, and other quality control mechanisms may not be reflected in this document. Changes may have been made to this work since it was submitted for publication. A definitive version was subsequently published in Electrochimica Acta, Vol. 101 (2013). DOI: 10.1016/j.electacta.2012.11.062 


\section{Chronoamperometric Response at Nanoscale Liquid-Liquid Interface Arrays}

Masniza Sairi ${ }^{a, 1}$, Jörg Strutwolf ${ }^{b}$, Rowan A. Mitchell ${ }^{a}$, Debbie S. Silvester ${ }^{a, 1}$ and Damien W. M. Arrigan a,1,

${ }^{a}$ Nanochemistry Research Institute, Department of Chemistry, Curtin University, GPO Box U1987, Perth, Western Australia 6845, Australia

${ }^{b}$ Institute of Organic Chemistry, University of Tübingen, Auf der Morgenstelle 18, 72076 Tübingen, Germany

* Corresponding author. Tel.: +61-8-9266-9735; Fax: +61-8-9266-2300; Email: d.arrigan@curtin.edu.au

\section{Abstract}

In this work, potential step chronoamperometry (PSCA) was used to study the behaviour of arrays of nanoscale interfaces between two immiscible electrolyte solutions (nanoITIES). The nanoITIES arrays were formed at nanoporous silicon nitride membranes containing 400 nanopores in a hexagonal close-packed arrangement. Three membrane designs, with nanopore radii of 75, 50 and $17 \mathrm{~nm}$, were studied by ion-transfer of tetrapropylammonium cations across the nanopore array-supported water|1,6-dichlorohexane interface. The cell time constants and charging times were determined prior to experimental PSCA. The three membrane designs studied exhibited charging times in the range of $0.08 \mathrm{~s}$ to $0.46 \mathrm{~s}$, with the smallest pore configuration (17 $\mathrm{nm}$ radius) exhibiting the longest charging time. The experimental steady-state currents were $30-50 \%$ lower than of the calculated inlaid disc model currents, due to diffusion zone overlap at adjacent interfaces. The three

\footnotetext{
${ }^{1}$ ISE member
} 
nano-interface arrays studied also showed response times of $6 \pm 1 \mathrm{~s}$, being the time required to reach $95 \%$ of the steady-state current.

Keywords: nanoITIES arrays, silicon nitride membranes, potential step chronoamperometry, charging time, response time.

\section{Introduction}

Electrochemistry at the interface between two immiscible electrolyte solutions (ITIES) [1] offers the possibility for detection of non-redox active ions, which cannot be detected at solid |liquid (electrode | solution) interfaces [2]. The detected current arises as a result of the applied potential-induced movement of ions across the liquid | liquid interface, in accordance with the ions' Gibbs energy of transfer. It affords a level of selectivity to the detection process, just as the applied potential does at solid | liquid interfaces [1-3]. Electrochemistry at micro- and nano-scale ITIES creates prospects for improved analytical sensitivity as a result of greater diffusional flux and often smaller iR drop [2, 4]. Recently, developments in electrochemical detection based on ion transfer across the ITIES have focussed on the detection and determination of small molecules and macromolecules of biological importance such as peptides [5], proteins [6], drugs, neurotransmitters and food additives [2].

To date, nano-scale ITIES (nanoITIES) have been prepared in two ways: (a) those supported at the tip of a single [7-9] or dual $[9,10]$ nanopipette (producing single or double nanoITIES) and (b) those produced by placing nanoporous materials containing geometrically irregular or regular pore arrays at the ITIES [11]. Track-etched polyester $[12,13]$ and $\gamma$-alumina ultrafiltration membranes [14] with high pore densities have been used to form irregular nanoITIES arrays, while silicon 
nitride membranes prepared by electron-beam lithography and chemical etching methods $[11,15]$ were used to form regular nanolTIES arrays. NanolTIES can offer benefits comparable to those experienced at nanoelectrode arrays, such as enhanced mass transport (due to radial diffusion), decreased charging current and decreased impact of solution resistance $[11,16]$. NanoITIES studies reported by Rimboud et al. [15], Liu et al. [4] and Shao and Mirkin [7] focussed on ion sensing using cyclic voltammetry as a technique. However, there is little information published on the chronoamperometric response at nano-interface arrays [13] despite numerous studies reporting the chronoamperometric response at microelectrochemical devices, e.g. micropipette-based ITIES [17-20], microdisc electrodes [21-24], micropore-based ITIES [13] and microarray electrodes [25].

Potential step chronoamperometry (PSCA) involves stepping the potential applied across the interface from a region where no Faradaic process occurs to a potential where a Faradaic process occurs and is mass-transport controlled. The resulting current is monitored as a function of time. This method generates high charging currents at short timescales, which decay exponentially with time. PSCA is widely used in the determination of diffusion coefficients and characterisation of electrodes and electrode reactions [26]. It also enables the determination of response time, a crucial parameter for chemical sensor applications. The response time of a chemical sensor can be defined as the time for the sensor signal to reach $95 \%$ of its final value $t_{95 \%}$ [27]. For PSCA at a nanolTIES, we can define the response time as the time needed to reach a current that is $95 \%$ of the steady-state current.

At electrified interfaces, two types of processes occur, Faradaic and nonFaradaic, and both contribute to the overall current, with Faradaic processes being of 
primary interest for sensing applications. At the ITIES, the Faradaic process is associated with the transfer of a charged species (ions, electrons or both) between the two liquid phases. Usually, in an amperometric or voltammetric sensing system, the non-Faradaic current associated with the charging of the interfacial capacitance is minimized. In designing or analysing an electrochemical experiment, consideration must be taken of the charging of the double layer at the electrochemical interface and its combination with the uncompensated resistance of the cell [28]. The Faradaic response for fast electrochemical or chemical reactions is restricted by this charging process [29].

The $R C$ time constant or cell time constant characterizes the timescale for the charging process and is generally modelled on the basis of a resistor and a capacitor in series $[28,30]$. This constant (which then leads to calculation of the charging time) has been reported by various researchers [18, 25, 31, 32]. Nirmaier and Henze reported that the cell time constant for establishing the electrode potential at the electrode surface varies linearly with the electrode surface radius for disk-shaped electrodes. Therefore, when a potential step is applied at a microelectrode, the charging process is much faster than for a millimetre-sized electrode [25].

However, this was found not to be the case when dealing with some liquid | liquid microinterface. PSCA experiments by Yuan et al. [18], Beattie et al. [31] and Shao and Mirkin [32] reported that the lower time scale (or charging time) for carrying out potential step experiments at a microITIES is crucially restricted by the large resistance at the narrow microhole of polymer membranes or glass micropipettes used to form such microlTIES. Comparison of the charging time of a water |1,2dichloroethane (DCE) microinterface with a metal | water microinterface of the same size showed that the charging time of the former was nearly three orders of 
magnitude greater than that of the latter [18]. For example, a cell time constant $R_{u} C_{d l}$ value of $80 \mu \mathrm{s}$ [18] was reported for a $10 \mu \mathrm{m}$ diameter water $\mid$ DCE interface with an uncompensated resistance, $R_{u}$ of $10 \mathrm{M} \Omega[31,32]$ and double-layer capacitance, $C_{d l}$ of $8 \mathrm{pF}$ [33]. Taking $\sim 5 R_{u} C_{d l}[18,30]$ as the fully established potential step, resulted in a charging time of $400 \mu \mathrm{s}$ [18]. The corresponding charging time for a metal | water interface of the same dimensions was $0.85 \mu$ s [30].

Numerous analytical expressions have been developed for analysing PSCA transients. For planar diffusion, the current-time transient is described by the Cottrell equation

$I=\frac{n F A c \sqrt{D}}{\sqrt{\pi t}}$

where $I$ is the diffusion-limited current, $n$ is the stoichiometric number of electrons involved in an electrode reaction, $F$ is Faraday's constant, $A$ is the electrode surface area, and $c, D$ and $t$ are, respectively, the bulk concentration of the electroactive species, the diffusion coefficient and the time [34].

At a microelectrode where convergent diffusion dominates, current-time expressions have been developed by Shoup and Szabo [35] and by Mahon and Oldham [36, 37]. The latter are the most exact closed-form expressions to describe the current-time transient, with a maximum error of $0.02 \%$. For short times, the expression is

$\frac{I(t)}{n F r D c}=\pi\left((\pi \tau)^{-1 / 2}+1+\frac{1}{2}\left(\frac{\tau}{\pi}\right)^{1 / 2}-0.12003 \tau+0.013273 \tau^{3 / 2}\right)$

and for times greater than $1.281 r^{2} / D$ the expression is

$$
\begin{aligned}
& \frac{I(t)}{n F r D C}=\pi\left(\frac{4}{\pi}+8 \pi^{-5 / 2} \tau^{-1 / 2}+8.9542 \times 10^{-3} \tau^{-3 / 2}-2.5664 \times 10^{-4} \tau^{-5 / 2}-2.2312 \times\right. \\
& \left.10^{-4} \tau^{-7 / 2}+2.7628 \times 10^{-5} \tau^{-9 / 2}\right)
\end{aligned}
$$


where $r$ and $\tau$ are, respectively, the electrode radius and the dimensionless time parameter.

$\tau=\frac{D t}{r^{2}}$

Equations (1), (2) and (3) can be readily applied to the ITIES, with $n$ replaced by the charge number of the transferring ion, $z$.

In this report, we present characterisation of the behaviour of nanoITIES arrays, formed at geometrically regular silicon nitride nanoporous membranes, by PSCA. The aim of the work was to evaluate the response times of these membranesupported ITIES so as to determine whether the radial diffusion advantage of nanoITIES was coupled with a fast response. The electrochemical transfer of the tetrapropylammonium cations $\left(\operatorname{TPrA}^{+}\right)$across the water | 1,6-dichlorohexane (DCH) interface was chosen as a model system. PSCA has not been applied previously to these nanoITIES arrays and can possibly reveal information about mass transport effects as well as the response time of the array, both of which may be useful in designing new arrays and sensors or detection systems based on them.

\section{Experimental}

\subsection{Reagents}

All reagents were obtained from Sigma Aldrich, Australia, and were used as received, unless stated otherwise. All aqueous solutions were prepared using deionized water with resistivity of $18 \mathrm{M} \Omega \mathrm{cm}$ from a Milli-Q water purification system (Millipore Pty Ltd, North Ryde, NSW, Australia). The organic phase solvent was 1,6dichlorohexane $(\mathrm{DCH})$. Both the aqueous and organic phase solvents were mutually pre-saturated prior to experiments. The supporting electrolytes in the aqueous and organic phases were $0.01 \mathrm{M}$ lithium chloride $(\mathrm{LiCl})$ and $0.01 \mathrm{M}$ 
bis(triphenylphosphoranylidene)ammonium tetrakis(4-chlorophenyl)borate (BTPPATPBCI), respectively. The organic electrolyte salt was prepared by metathesis of bis(triphenylphosphoranylidene)ammonium chloride (BTPPACl) and potassium tetrakis(4-chlorophenyl)borate (KTPBCl). The organic reference solution was $0.01 \mathrm{M}$ BTPPACl dissolved in aqueous $0.01 \mathrm{M} \mathrm{LiCl}$. The model analyte studied was the chloride salt of tetrapropylammonium $\left(\operatorname{TPrA}^{+}\right)$in $0.01 \mathrm{M} \mathrm{LiCl}$ in $\mathrm{DCH}-$ saturated water.

\subsection{Preparation of nano-interface arrays}

Arrays of nano-ITIES were formed at a water | DCH interface using silicon nitride $\left(\mathrm{Si}_{3} \mathrm{~N}_{4}\right)$ nanopore array membranes (100 nm thick) on a silicon chip support, which were fabricated at Tyndall National Institute, University College Cork, Cork, Ireland $[11,38]$. The silicon chips $(5 \mathrm{~mm} \times 5 \mathrm{~mm})$ containing the nanopore array membranes were sealed onto the lower orifice of cylindrical borosilicate glass tubes $(2.5 \mathrm{~mm}$ inner and $4 \mathrm{~mm}$ outer diameters) using silicone rubber (Selleys, Australia and New Zealand) and allowed to cure for 72 hours before use. Three sizes of nanopore radius were used in this study ( $r=75,50$ or $17 \mathrm{~nm}$ ). Each membrane contained 400 pores in a hexagonal close-packed arrangement with pore centre-to-centre separations, $r_{c}$, of 20 -times the pore radius, $r,\left(r_{c}=20 r\right)$. Scanning electron microscopy (SEM) micrographs of these nanopore arrays have been reported previously $[11,15,38]$.

\subsection{Experimental procedure}

A two-electrode electrochemical cell was employed in which both $\mathrm{Ag} \mid \mathrm{AgCl}$ electrodes served as reference and counter electrodes in their respective phases. 
The borosilicate glass tube, to which the membrane was affixed and contained approximately $50 \mu \mathrm{L}$ of the organic phase and $250 \mu \mathrm{L}$ of the organic reference solution, was immersed in $3 \mathrm{~mL}$ of the aqueous phase solution in a $10 \mathrm{~mL}$ glass beaker. Prior to the injection of a concentrated solution of $\operatorname{TPrA}^{+}$into the aqueous phase with a micropipette to achieve the required analyte concentration, a background voltammogram was run over a wide potential range to establish the limits of the available potential window. A sequence of three background voltammograms was then recorded over a potential range which encompassed the transfer of $\mathrm{TPrA}^{+}$when it was subsequently added to the aqueous phase. A quiet time of $5 \mathrm{~s}$ at the initial applied potential was implemented prior to each scan to stabilize and minimize the background charging current. The electrochemical cell can be summarized as follows:

$\mathrm{Ag}|\mathrm{AgCl}| x$ mM TPrACl $+0.01 \mathrm{M} \mathrm{LiCl}_{W}||$

$0.01 \mathrm{M} \mathrm{BTPPATPBCl}_{D C H} \mid 0.01 \mathrm{M} \mathrm{BTPPACl}$ in $0.01 \mathrm{M} \mathrm{LiCl}_{\mathrm{W}}|\mathrm{AgCl}| \mathrm{Ag}$

(where $x$ is the concentration of TPrACl in the aqueous phase).

The electrochemical methods PSCA and cyclic voltammetry (CV) were applied using an Autolab PGSTAT 302N (Metrohm, The Netherlands) interfaced to a personal computer running the Nova 1.7 software package supplied with the instrument. The voltammetric sweep rate was $10 \mathrm{mV} \mathrm{s}^{-1}$ (75 and $50 \mathrm{~nm}$ radii pores) or $5 \mathrm{mV} \mathrm{s}^{-1}$ (17 $\mathrm{nm}$ radius pores). No automatic positive feedback compensation was applied during PSCA and CV. The electrochemical cell was placed in a Faraday cage to minimise electrical noise. For PSCA, the steady-state current $I_{S S}$ was taken as the average of the final ten current points of the $10 \mathrm{~s}$ transient, taken at $10 \mathrm{~ms}$ intervals, corresponding to the final $100 \mathrm{~ms}$ of the measurement. 


\section{Results and discussion}

\subsection{Cyclic voltammetry of $\operatorname{TPrA}^{+}$transfer at the nano-ITIES array}

Initially, ion transfer across the nanoITIES array was characterised using CV so that initial and step potentials could be selected for the PSCA study. TPrA ${ }^{+}$was chosen as the model analyte ion and aqueous phase concentrations between 20 and $100 \mu \mathrm{M}$ in increments of $20 \mu \mathrm{M}$ in a background of $0.01 \mathrm{M} \mathrm{LiCl}$ were employed. Prior to the addition of analyte, a CV of the background electrolyte solutions was recorded, so that background-subtracted voltammograms could be obtained. Figure 1(a) shows a typical set of background-subtracted voltammograms (forward scans only) obtained at a nanolTIES array with interface radius of $50 \mathrm{~nm}$. This figure corresponds to the transfer of $\operatorname{TPrA}^{+}$from aqueous to organic phase. These CVs show that the current rose steadily with applied potential up to the switching potential and that no true steady-state current plateau was reached in the diffusion-limited region, in agreement with previous reports [15] for the transfer of tetraethylammonium cation across the water | liquid DCH interface. From Figure $1(\mathrm{a})$, it can be seen that the current in the diffusion-limited region (above 0.6 V) increases with the aqueous phase concentration of $\operatorname{TPrA}^{+}$, with a linear relationship as shown by the inset graph of current versus concentration of transferring ion. In this case, the experimental currents were determined at a potential ca. $200 \mathrm{mV}$ positive of the foot of ion transfer wave [15]. Concurrently, CV was used in the determination of the step potentials for chronoamperometric experiments in Section 3.3 in which the potential was stepped from $0.2 \mathrm{~V}$ to $0.6 \mathrm{~V}$.

\subsection{Estimation of charging time}


Determination of the cell time constant, $R_{u} C_{d l}$ allows in turn the determination of the charging time. Execution of a PSCA measurement at times shorter than this charging time will not produce useful data [30]. In fact, the minimum time for full establishment of a potential step requires $\sim 5 R_{u} C_{d l}$ [30] as applied by Yuan et al. [18] in their micropipette-based ITIES study. Normally, the step must last at least $10 R_{u} C_{d l}$ which includes time for recording data beyond the time required for establishment of the potential step [30]. The size of the electrode governs the cell time constant and consequently the charging time. In this study, the charging time was taken as $5 R_{u} C_{d l}$ [30]. In order to estimate the cell time constant and the charging time, values of the uncompensated resistance and the double layer capacitance are needed.

The main source of uncompensated resistance in ITIES electrochemical cells is the low conductivity of the organic electrolyte solution. Katano and Senda [39] reported a conductivity of $\kappa=49 \mu \mathrm{S} \mathrm{cm}^{-1}$ for $\mathrm{DCH}$ with tetraoctylammonium tetrakis(4-chlorophenyl)borate as an electrolyte. It was shown [40-42] that the conductivity was similar regardless of the electrolyte types, based on a study with a series of electrolytes in DCE. Therefore, it is reasonable to assume a value of $49 \mu \mathrm{S}$ $\mathrm{cm}^{-1}$ for the conductivity of the organic phase employed here. For comparison, the conductivity of aqueous $0.01 \mathrm{M} \mathrm{LiCl}$, used as the aqueous electrolyte, is $1073 \mu \mathrm{S} \mathrm{cm}^{-}$ ${ }^{1}$ [43]. In the organic phase, the resistance will be due to the distance between the tip of the counter/reference electrode and the orifice of the pore ("bulk solution" resistance, $R_{b}$ ) and to the resistance within the pore (pore resistance, $R_{p}$ ). Both $R_{b}$ and $R_{p}$ will contribute to the uncompensated resistance.

The pore resistance, $R_{p}$, is given by

$$
R_{p}=\frac{l}{\pi r^{2} \kappa}
$$


where $l$ is the pore length $[42,44]$. Each pore is assumed to be filled by the organic electrolyte solution, resulting in inlaid nanoITIES arrays $[11,15]$. The pore resistance increases with an increase in the pore length and reduction in pore radius. The interface cross-sectional region in direct electrical contact with the aqueous or organic phase is defined by the diameter of the nanopore. If the individual pores in the array behave like resistors in parallel, the total inverse resistance value is obtained by multiplying the inverse resistance of a single pore by the number of pores in the array $[13,42]$. The individual pore resistances for the 75,50 and $17 \mathrm{~nm}$ pore radii are $1.15 \times 10^{9} \Omega, 2.60 \times 10^{9} \Omega$ and $22.48 \times 10^{9} \Omega$, respectively, calculated using equation 5 . Thus, the total pore resistance across the three membranes studied are in the range 2.89 to $56.19 \mathrm{M} \Omega$, with the smaller nanopore radius membranes exhibiting larger resistances. These results agree with the resistance estimation by Strutwolf et al. [42] of $103 \times 10^{5} \Omega$ per pore for $25 \mu \mathrm{m}$ radius pore and $650 \times 10^{5} \Omega$ per pore for a $10 \mu \mathrm{m}$ radius pore. The resistances were higher at nanoscale pores. Additionally, the total pore resistance of the membranes with micron-sized pores $(r=25 \mu \mathrm{m})$ increased from $9.8 \times 10^{4} \Omega$ to $3.4 \times 10^{6} \Omega$ with a decreased number of pores (from 105 to 3 pores) [42].

The dimension of the counter/reference electrode is much larger than the critical dimension of the pore $(r)$, and the distance between the pore and the tip of the reference/electrode is in the millimetre range and therefore much bigger than $r$. Under these conditions, the bulk resistance can be calculated from [45]

$R_{b}=\frac{1}{4 \pi r \kappa}$

For the 17,50 and $75 \mathrm{~nm}$ radius pores, $R_{b}$ values of $0.95 \times 10^{9} \Omega, 0.32 \times 10^{9} \Omega$, and $0.22 \times 10^{9} \Omega$ are calculated for the individual pores in the arrays. Again, the resistance established between each individual pore of an array and the tip of the 
reference/counter electrode acts like a resistor in parallel with the other bulk solution resistors, but in series with the pore resistance of the same pore (given by Eq. (5)). So the total bulk resistances for the three arrays are $0.55,0.81$ and $2.37 \mathrm{M} \Omega$ with decreasing pore radius. The total or uncompensated resistance is $3.42,7.30$ and 58.57 $\mathrm{M} \Omega$ for the 75, 50 and $17 \mathrm{~nm}$ arrays, respectively (Table 1). Since the pore resistances scales with $r^{-2}$, while bulk resistance scales with $r^{-1}$, the influence of the pore resistance on the uncompensated resistance is more pronounced the smaller the pore radius. For the $75 \mathrm{~nm}$ pore array, the bulk resistance contributes 19 $\%$ to the total resistance, while for the $17 \mathrm{~nm}$ array the contribution is $4 \%$.

In this report, the experimental capacitance $C_{\text {expt }}$ was determined by $\mathrm{CV}$ of the electrolyte system in the absence of ion transfer, as obtained by

$C_{\text {expt }}=I_{c} / 2 v$

where $I_{c}$ and $v$ are the total charging current and scan rate, respectively [46]. The experimental capacitance is used here because, as will be apparent below, the double layer capacitance of the ITIES is but one contribution to the overall capacitance measured. The charging current was determined from CVs of the electrolyte solutions at a potential where no ion transfer occurs (Figure 1(b)). All membranes studied exhibited experimental capacitance values in the nanoFarad regime, ranging from 1.57 to $4.83 \mathrm{nF}$ (Table 1 ).

Assuming for the moment that the experimental capacitance is solely due to the capacitance of the nanoITIES, the specific capacitance or capacitance per unit area $C_{\text {expt }}^{0}$ can be determined. The experimental capacitance was divided by the total pore cross-sectional area, resulting in the experimental specific capacitances $C_{\text {expt }}^{0}$ with the average value ranging between 630 and $4320 \mathrm{~F} \mathrm{~m}^{-2}\left(0.63 \times 10^{5}\right.$ and $4.32 \times 10^{5}$ $\mu \mathrm{F} \mathrm{cm}^{-2}$ ) (Table 1). 
For comparison purposes, the capacitance per unit area from the literature $C_{\text {lit }}^{0}$ was obtained by dividing the literature value $C_{l i t}$ by the nanopore array crosssectional area. Trojanek et al. [33] reported a capacitance value of $0.08 \mathrm{~F} \mathrm{~m}^{-2}$ for the interface between $0.1 \mathrm{M} \mathrm{LiCl}$ in water and $5 \mathrm{mM}$ BTPPATPBCl in DCE. Assuming this value as the typical specific interfacial capacitance at the ITIES, and applying it to our system, showed that our experimental values were about four orders of magnitude higher (Table 1). These results indicate that capacitance effects in addition to that of the ITIES are present. A further source of capacitance is the $\mathrm{Si}_{3} \mathrm{~N}_{4}$ membrane. In the experimental set-up, the $\mathrm{Si}_{3} \mathrm{~N}_{4}$ membrane can be considered as a dielectric $\left(\varepsilon_{r}=7.2\right)$ membrane sandwiched between two conductors (electrolyte solutions) whose capacitance can be described by the parallel plate model:

$C=\frac{\varepsilon_{r} \varepsilon_{0} A}{l}$

where $\varepsilon_{0}=8.85 \times 10^{-12} \mathrm{~F} \mathrm{~m}^{-1}$ is the vacuum permittivity, $A=500 \times 500 \mu \mathrm{m}^{2}$ is the area of the membrane (neglecting the pores) and $l=100 \mathrm{~nm}$ is the membrane thickness $[47,48]$. The calculated capacitance is $0.16 \mathrm{nF}$. This is one order of magnitude lower than the overall capacitance of the systems determined experimentally in this study (Table 1). The difference between the experimentallydetermined capacitances and the calculated capacitance for the silicon nitride membrane suggests that additional capacitive components are present, in addition to that of the membrane material. Further study on the system of capacitances is necessary and is under way using electrochemical impedance spectroscopy (EIS). Scanlon et al. [11] investigated the capacitance between two electrolyte solutions separated by a $\mathrm{Si}_{3} \mathrm{~N}_{4}$ membrane without pores and compared this with a nanolTIES array containing 23 nano-interfaces, with $r \sim 45 \mathrm{~nm}$. In both cases, a blank electrolyte solution system was applied (i.e., in the absence of a transferring ion). 
The blank CVs obtained were virtually the same, showing that the overall capacitance has only a marginal contribution from the nanoITIES.

By combining the experimental capacitance and the uncompensated resistance, the cell time constants for the three membrane designs employed here were obtained. The three membrane array designs exhibited (Table 1) cell time constants and charging times in the range of $0.017 \mathrm{~s}$ to $0.092 \mathrm{~s}$ and $0.08 \mathrm{~s}$ to $0.46 \mathrm{~s}$, respectively, with the nanoITIES array with the smallest interface radius $(17 \mathrm{~nm}$ radius) exhibiting the largest pore resistance and consequently the longest charging time. The charging time was observed to be more influenced by the resistance than the capacitance, which can be seen by the resistance range (from 3.42 to $58.57 \mathrm{M} \Omega$ ) being broader than the capacitance range (1.57 to $4.83 \mathrm{nF})$. In all experimental current transients reported below, data from times shorter than the charging times were omitted.

Data from this study were compared to data from Yuan et al. [18] at a single microlTIES formed at $5 \mu \mathrm{m}$ radius micropipette, since the total nanolTIES array may behave as microITIES. The principle underlying this assumption was that an array of nanoelectrodes eventually behaves as if the entire array were a single electrode of the same interfacial surface area, with its equivalent properties $[11,15,49]$. If the charging time was taken as $5 R_{u} C_{\text {expt }}$, the microlTIES exhibited a charging time of 0.4 ms, which was approximately three orders of magnitude faster than at the nanointerface arrays (Table 1). Similarly, use of the literature value for the liquid | liquid interfacial capacitance [33] together with the estimated uncompensated resistance for the nanoITIES arrays studied here, gives values of charging time between $0.14 \mathrm{~ms}$ to $2.34 \mathrm{~ms}$, which are again much shorter than those obtained using the experimentally-determined capacitances. The comparison of our results 
with literature data indicates that the combination of the large resistance of the nanopore system with the as-yet undetermined capacitance due to the presence of the silicon nitride membrane contributes to long charging times at the nanoITIES array employed here. As a result the application of methods such as differential pulse voltammetry (DPV) and square wave voltammetry (SWV) to characterise and exploit the ion transfer process at the nano-interface arrays may not produce useful data as these methods operate in millisecond and sub-millisecond time domains [50].

\subsection{Chronoamperometry at the nanoITIES array}

The variation of the current response with time under potentiostatic control across the nanopore array-supported water $\mid \mathrm{DCH}$ interface was recorded using $\operatorname{TPrA}^{+}$as the model analyte for these PSCA studies. Chronoamperograms were recorded at five different concentrations of TPrACl ranging from 20 to $100 \mu \mathrm{M}$ in steps of $20 \mu \mathrm{M}$. The initial potential, $E_{1}(+0.2 \mathrm{~V})$, was chosen where no current flows, while the step or transfer potential, $E_{2}(+0.6 \mathrm{~V})$, was chosen where the ion transfer process occurs and was always in the region beyond the lower limits of mass transport control, i.e. within the "sloping" limiting current region of Figure 1(a). The potential was held for $20 \mathrm{~s}$ at the initial potential and $10 \mathrm{~s}$ at the step potential. By holding at the initial potential for 20 seconds, ions present in the organic phase were back extracted into the aqueous phase and hence regenerating the initial conditions.

Figure 2(a) shows the background-subtracted chronoamperograms for $100 \mu \mathrm{M}$ TPrACl in the aqueous phase, using nanolTIES arrays patterned by 75,50 and 17 $\mathrm{nm}$ radii pore arrays. The data at shorter times than the calculated charging times (Table 1) were excluded from the figures. It can be seen that the experimental 
transient obtained at the nanoITIES arrays decayed to a steady-state current as suggested by Equations (2) and (3), as opposed to the $t^{-1 / 2}$ dependence expected for Cottrellian behaviour. Two different time regimes can be observed at the nanoITIES arrays from these PSCA experiments. Firstly, at short times, a rapid decrease of current is seen and, secondly, a steady-state current is observed at long times [51]. However, note that currents at times shorter than the charging times are omitted from Figure 2(a). Nevertheless, there is a substantial current decrease and given that the currents were background-subtracted, these decreasing currents must be due to mass transport of the $\operatorname{TPrA}^{+}$cations to the nanolTIES arrays. Figure 2(b) shows examples of background, ion-transfer and background-subtracted ion transfer current transients. The background-subtraction removed the background-charging process from the current transient, and demonstrated that the short-time region is mainly influence by the charging current but this charging current has a minimal impact on the longer-time diffusion-controlled current.

Chronoamperometry allows the determination of the response time, an important parameter in sensor applications, where a fast response is often required. In chemical sensors, the response time may be typically defined as the time taken to reach a signal that is $95 \%$ of the final signal value [27]. Similarly, in PSCA, the response time can be defined as the time taken for the current to reach $95 \%$ of the steady-state value, given by

$I_{95 \%}=1 /\left(0.95 \times I_{S S}\right)$

Theoretically, the smallest membrane pore radius $(17 \mathrm{~nm})$ should achieve steadystate faster as compared to the larger pore membranes, because of the dominance of radial diffusion [16] . However, the results obtained from Figure 2(a) clearly show that the response was dominated by the electrical properties of the membrane. This 
can be seen in the similar response times for the three nanoITIES arrays studied at 6 $\pm 1 \mathrm{~s}$ (Table 1). The raw experimental response times were all in the range of 5 to 7 s, irrespective of the pore size used in the experiments and no apparent trend was observed as the pore size was changed. The long response times obtained can be attributed to the highly resistive and capacitive membranes used to pattern the nanoITIES and may render them unfavourable for use in rapid-response chemical and biochemical sensor applications.

In section 3.2, an assumption was made that the interfaces studied were inlaid. Thus, the formula to calculate the steady-state current at a single interface for an ion transferring from the aqueous to the organic phase is given by the Saito equation, $I_{s S}=4|z| F D c r$

where $I_{S S}$ is the steady-state current (or limiting current) [52]. The steady-state current is a linear function of the concentration and radius $[15,24,52]$. To obtain the total current of the array, the steady-state current calculated for one pore must be multiplied by the number of pores $N_{p}$, in this case, 400 . By applying this expression for a fixed analyte concentration (e.g. $100 \mu \mathrm{M}$ of $\operatorname{TPrA}^{+}$), the calculated steady-state currents are $0.80,0.53$, and $0.18 \mathrm{nA}$ for the nanolTIES arrays based on pore radii of 75,50 and $17 \mathrm{~nm}$, respectively, which varied linearly with the radius. The average of the last 10 current points from the PSCA transient was selected as the experimental steady-state current (i.e. the final $100 \mathrm{~ms}$ of the current transient).

As shown in Figure 3(b), the experimental steady-state currents were lower than the calculated inlaid disc currents (equation (10)), in agreement with previous studies $[15,49]$. They were lower by $30-50 \%$ of the theoretical currents, while Rimboud et al. [15] reported the experimental limiting currents were ca. $50 \%$ of the calculated currents (equation (10)). Those studies demonstrated that diffusion zone overlap, 
which occurred at adjacent interfaces in the array, lead to non-independent diffusion to each interface in the array, and resulted in the difference between experimental and calculated steady-state currents. Radial diffusion dominates the interfaces at the edge of the arrays while linear diffusion governs the interfaces within the arrays [11]. Particularly, the electrode-to-electrode separation (in this study equivalent to the pore-to-pore separation) and the number of nanoelectrodes (equivalent to the number of nanopores or nano-interfaces) are the key parameters that influenced the measured currents. Although the lower currents recorded could be associated with recessed rather than inlaid interfaces, previous studies have indicated that the interfaces are indeed inlaid and thus implicating diffusion zone overlap as the reason for lower currents $[11,15]$.

Figure 4 represents the experimental, Cottrellian and Mahon and Oldham current transients for transfer of $100 \mu \mathrm{M}$ TPrACl across the $75 \mathrm{~nm}$ radius nanolTIES array. The Cottrellian current response in the time range was so low that it appears as zero in the figure. However, the inset magnified view of the current transient shows that it approaches zero, but does not reach zero. In addition, the change in current over the timescale is too small to be seen in the comparison figure. For the Mahon and Oldham currents, the steady-state current is portrayed on the figure and is larger than the experimental current, for the reasons discussed above. Additionally, the transient response is not visible on the current scale displayed, but the inset clearly shows that this current decays rapidly with time to achieve the steady state value. For the Mahon and Oldham current, only the long-time expression (equation (3)) was applied, as the short time expression was too short $(t<0.01 \mathrm{~ms})$ for the charging times of the experimental arrangements used here. The Mahon and Oldham expression is accurate provided that the uncompensated resistance is negligible 
[37], in contrast with large resistance results from this study. The large resistance has a retarding effect on the response time, illustrated by the slower decrease of experimental current than this expression (equation (3)). However, the steady-state current at longer times will not be affected by the large resistance. Note that the long-term expression of Mahon and Oldham gives the same value as the Saito equation for the steady-state current.

Comparison of the experimental currents with the Cottrell and Mahon and Oldham expressions showed non-agreement with either model, in terms of both timescale of response and magnitude of current. The time-dependent currents will obey the Cottrell equation only if the electroactive interface is subjected to planar diffusion [53]. On the other hand, at microlTIES, convergent diffusion dominates. In the case of a nanoITIES array with overlapping diffusion zones at adjacent nanoITIES, as is the situation here, the array may behave like a single microITIES of the same geometric parameters as those of the total nano-array [49]. In such a case, the current transient may be expected to follow the trend of a single microlTIES. The transition to a steady-state can be observed in Figure 4. The steady-state current was smaller than expected because of diffusion zone overlap, i.e. the diffusional transport to the individual pores was reduced (compared to the case of a single pore membrane) due to competing diffusion zones around the pores. The time required to achieve the steady-state current was also slower than predicted by the Mahon and Oldham equation (equation (3)).

The impact of solution resistance of the $I R$ drop can be considered as follows. If the current at an electrode is under diffusion control, the potential drop is expressed by $[54,55]$

$I_{S S} R=\frac{n F D\left(c^{b}-c_{S}\right)}{\kappa}$ 
where $c^{b}$ and $c_{s}$ are the bulk and surface concentrations and the other parameters are as previously defined. Note that the surface concentration is zero in the region of the steady-state limiting current. The number of electrons involved in the electrode reaction, $n$, is equivalent to $z$, the charge number of the transferring ion. Equation (11) was derived under the assumption that the size of the counter electrode and the distance between it and the working electrode (here the ITIES established at the orifice of the nanopore) are much bigger than the size of the working electrode (i.e. the pore radius) and the diffusion layer established during an experiment. Furthermore, an excess of supporting electrolyte is present. These conditions are met in the present experiments. Using a concentration of $100 \mu \mathrm{M}$, a diffusion coefficient of $10^{-6} \mathrm{~cm}^{2} \mathrm{~s}^{-1}$ and a conductivity of $1073 \mu \mathrm{S} \mathrm{cm}$-1 for the aqueous electrolyte (0.01 M LiCl) [43] results in an $I_{s s} R$ drop of $0.01 \mathrm{mV}$ in the limiting current region, a value which is negligible. However, this approach does not take into account the resistance of the organic phase, which is a combination of the pore resistance and the solution resistance. A rough estimation of the potential drop can be made as follows. Previously, the individual pore resistance was calculated to be $2.6 \times 10^{9} \Omega$ for pores with a radius of $50 \mathrm{~nm}$. The limiting current of the pore array (Figure 2(b)) is ca. $0.4 \mathrm{nA}$ which gives a current of approximately $1 \mathrm{pA}$ for an individual pore, resulting in $I_{s S} R \approx 3 \mathrm{mV}$. The potential drop is likely to be bigger due to the additional solution resistance. These values of potential drop will interfere with quantitative analysis of transfer kinetics. However, the steady-state limiting current will not be affected and therefore a potential drop of this magnitude might be negligible for electroanalytical purposes based on analysis of limiting currents.

Finally, to study the agreement between currents obtained from the forward scans of cyclic voltammetry experiments with the currents obtained from PSCA, the 
step potential was increased in increments of $0.05 \mathrm{~V}$. At long times $(t=10 \mathrm{~s})$, the PSCA data agreed well with the voltammetry data, as shown in Figure 5. As discussed in Section 3.1, no true limiting current plateau is reached when implementing voltammetry at the nanoITIES arrays. Nevertheless, current data obtained from potential-dependent and time-dependent experiments are in excellent agreement.

\section{Conclusions}

Chronoamperometry of $\operatorname{TPrA}^{+}$ion transfer was performed at the water | 1,6dichlorohexane nano-interface array using three different nanopore array membrane designs (nanopore radii of 75, 50 and $17 \mathrm{~nm}$ ). An assumption that the interfaces were inlaid was made, in which liquid organic phase filled the silicon nitride nanopores. The nanoITIES arrays exhibited prolonged charging times, hence the Faradaic current can be measured only at long times (timescale of seconds). The system demonstrated greater capacitances than predicted using literature values for the capacitance of the ITIES, indicating that capacitances additional to the interfacial liquid | liquid capacitance have an impact on the behaviour of these nanoporous membrane systems, such as the capacitance of the silicon nitride membrane employed. The resistances of the nanopores filled with organic phase electrolyte varied more with nanopore size than the overall system capacitance, indicating that the uncompensated resistances dominated the responses. The experimental current transients were not in agreement with either Cottrell or Mahon and Oldham expressions, and experimental steady-state currents were lower than predicted, indicating overlapping diffusion zones. However, the currents from chronoamperometry were in excellent agreement with those obtained from cyclic 
voltammetry. The response times (time to reach $95 \%$ of the steady-state current) of the silicon nitride membrane-based nanoITIES arrays to a potential step were in the region of $6 \mathrm{~s}$, indicating a slow response and the impact of nanopore resistance as well as membrane capacitance. The results presented here provide further characterisation of nanoITIES arrays and will be beneficial in the design of chemical and biochemical sensing systems.

\section{Acknowledgements}

This work was supported by Curtin University, the Western Australian Nanochemistry Research Institute (WANRI) (a WA State Centre of Excellence) and the Malaysian Agricultural Research and Development Institute (MARDI). The authors thank Tyndall National Institute, Cork, Ireland, for the gift of the nanopore array membranes.

\section{References}

[1] Z. Samec, Electrochemistry at the interface between two immiscible electrolyte solution, Pure Appl. Chem. 76 (2004) 2147.

[2] D.W.M. Arrigan, Bioanalytical detection based on electrochemistry at interfaces between immiscible liquids, Anal. Lett. 41 (2008) 3233.

[3] P. Vanysek, L.B. Ramirez, Interface between two immiscible liquid electrolytes: A review, J. Chil. Chem. Soc. 53 (2008) 1455.

[4] S. Liu, Q. Li, Y. Shao, Electrochemistry at micro- and nanoscopic liquid/liquid interfaces, Chem. Soc. Rev. 40 (2011) 2236.

[5] M.D. Scanlon, G. Herzog, D.W.M. Arrigan, Electrochemical detection of oligopeptides at siliconfabricated micro-liquid|liquid interfaces, Anal. Chem. 80 (2008) 5743.

[6] G. Herzog, W. Moujahid, J. Strutwolf, D.W.M. Arrigan, Interactions of proteins with small ionised molecules: Electrochemical adsorption and facilitated ion transfer voltammetry of haemoglobin at the liquid|liquid interface, Analyst 134 (2009) 1608.

[7] Y. Shao, M.V. Mirkin, Fast kinetic measurements with nanometer-sized pipets. Transfer of potassium ion from water into dichloroethane facilitated by dibenzo-18-crown-6, J. Am. Chem. Soc. 119 (1997) 8103.

[8] P. Sun, Z. Zhang, Z. Gao, Y. Shao, Probing fast facilitated ion transfer across an externally polarized liquid-liquid interface by scanning electrochemical microscopy, Angew. Chem., Int. Ed. 41 (2002) 3445.

[9] F.O. Laforge, P. Sun, M.V. Mirkin, Shuttling mechanism of ion transfer at the interface between two immiscible liquids, J. Am. Chem. Soc. 128 (2006) 15019. 
[10] B. Liu, Y. Shao, M.V. Mirkin, Dual-pipet techniques for probing ionic reactions, Anal. Chem. 72 (2000) 510.

[11] M.D. Scanlon, J. Strutwolf, A. Blake, D. lacopino, A.J. Quinn, D.W.M. Arrigan, Ion-transfer electrochemistry at arrays of nanointerfaces between immiscible electrolyte solutions confined within silicon nitride nanopore membranes, Anal. Chem. 82 (2010) 6115.

[12] R.A.W. Dryfe, B. Kralj, Voltammetric ion transfer in the presence of a nanoporous material, Electrochem. Commun. 1 (1999) 128.

[13] B. Kralj, R.A.W. Dryfe, Membrane voltammetry: The interface between two immiscible electrolyte solutions, Phys. Chem. Chem. Phys. 3 (2001) 5274.

[14] M. Platt, R.A.W. Dryfe, E.P.L. Roberts, Voltammetry with liquid/liquid microarrays:

Characterization of membrane materials, Langmuir 19 (2003) 8019.

[15] M. Rimboud, R.D. Hart, T. Becker, D.W.M. Arrigan, Electrochemical behaviour and voltammetric sensitivity at arrays of nanoscale interfaces between immiscible liquids, Analyst 136 (2011) 4674.

[16] D.W.M. Arrigan, Nanoelectrodes, nanoelectrode arrays and their applications, Analyst 129 (2004) 1157.

[17] Y. Yuan, S. Amemiya, Facilitated protamine transfer at polarized water/1,2-dichloroethane interfaces studied by cyclic voltammetry and chronoamperometry at micropipet electrodes, Anal. Chem. 76 (2004) 6877.

[18] Y. Yuan, L. Wang, S. Amemiya, Chronoamperometry at micropipet electrodes for determination of diffusion coefficients and transferred charges at liquid/liquid interfaces, Anal. Chem. 76 (2004) 5570.

[19] P. Beattie, A. Delay, H. Girault, Investigation of the kinetics of assisted potassium ion transfer by dibenzo-18-crown-6 at the micro-ITIES by means of steady-state voltammetry, J. Electroanal. Chem. 380 (1995) 167.

[20] Y.B. Liao, M. Okuwaki, F. Kitamura, T. Ohsaka, K. Tokuda, Normal pulse voltammetry for facilitated ion transfer processes across two immiscible liquid-liquid interfaces, Electrochim. Acta 44 (1998) 117.

[21] T. Hepel, J. Osteryoung, Chronoamperometric transients at the stationary disk microelectrode, J. Phys. Chem. 86 (1982) 1406.

[22] P.N. Bartlett, S.L. Taylor, An accurate microdisk simulation model for recessed microdisk electrodes, J. Electroanal. Chem. 453 (1998) 49.

[23] R. Ferrigno, P.F. Brevet, H.H. Girault, Finite element simulation of the chronoamperometric response of recessed and protruding microdisk electrodes, Electrochim. Acta 42 (1997) 1895.

[24] A.M. Bond, D. Luscombe, K.B. Oldham, C.G. Zoski, A comparison of the chronoamperometric response at inlaid and recessed disk microelectrodes, J. Electroanal. Chem. 249 (1988) 1.

[25] H.P. Nirmaier, G. Henze, Characteristic behavior of macro-, semimicro- and microelectrodes in voltammetric and chronoamperometric measurements, Electroanalysis 9 (1997) 619.

[26] M.C. Henstridge, R.G. Compton, Mass transport to micro and nanoelectrodes and their arrays: A review, Chem. Rec. 12 (2012) 63.

[27] R.W. Cattrall, Chemical Sensors, Oxford University Press Inc., New York, 1997, pp. 25.

[28] K.B. Oldham, The RC time "constant" at a disk electrode, Electrochem. Commun. 6 (2004) 210.

[29] J.C. Myland, K.B. Oldham, How does the double layer at a disk electrode charge?, J. Electroanal. Chem. 575 (2005) 81.

[30] A.J. Bard, L.R. Faulkner, Electrochemical Methods: Fundamentals and Applications, 2nd ed., John Wiley and Sons Inc., New York, 2001, pp. 216-218.

[31] P. Beattie, A. Delay, H. Girault, Investigation of the kinetics of ion and assisted ion transfer by the technique of ac impedance of the micro-ITIES, Electrochim. Acta 40 (1995) 2961.

[32] Y. Shao, M.V. Mirkin, Voltammetry at micropipet electrodes, Anal. Chem. 70 (1998) 3155.

[33] A. Trojanek, A. Lhotsky, V. Marecek, Z. Samec, Limited agreement between the interfacial tension and differential capacity data for the polarized water|1,2-dichloroethane interface, J. Electroanal. Chem. 565 (2004) 243. 
[34] F.G. Cottrell, Residual current in galvanic polarization, regarded as a diffusion problem, Z. Phys. Chem 42 (1903) 385.

[35] D. Shoup, A. Szabo, Chronoamperometric current at finite disk electrodes, J. Electroanal. Chem. 140 (1982) 237.

[36] P.J. Mahon, K.B. Oldham, The transient current at the disk electrode under diffusion control: A new determination by the Cope-Tallman method, Electrochim. Acta 49 (2004) 5041.

[37] P.J. Mahon, K.B. Oldham, Diffusion-controlled chronoamperometry at a disk electrode, Anal. Chem. 77 (2005) 6100.

[38] J.S. Ellis, G. Herzog, B. Glynn, D.W.M. Arrigan, Electrochemical characterization of regularlyaligned nanopore array membranes filled with electrolyte solutions and their use for detection of nucleic acid hybridization, ECS Trans. 35 (2011) 29.

[39] H. Katano, M. Senda, Voltammetry at 1, 6-dichlorohexane: water interface, Anal. Sci. 17 (2001) 1027.

[40] A.K. Kontturi, K. Kontturi, J.A. Manzanares, S. Mafe, L. Murtomäki, lon pairing in the analysis of voltammetric data at the ITIES: RbTPB and RbTPBCl in 1, 2-dichloroethane, Ber. Bunsenges. Phys. Chem. 99 (1995) 1131.

[41] K. Sawada, F. Chigira, Ion pair of tetraalkylammonlum picrates, J. Mol. Liq. 65/66 (1995) 265. [42] J. Strutwolf, M.D. Scanlon, D.W.M. Arrigan, Electrochemical ion transfer across liquid/liquid interfaces confined within solid-state micropore arrays - Simulations and experiments, Analyst 134 (2009) 148.

[43] P. Vanysek, Equivalent conductivity of electrolytes in aqueous solution, in: D.R. Lide (Ed.), CRC Handbook of Chemistry and Physics (Online), CRC Press, Boca Raton, FL, 2012, pp. 5.76.

[44] R.W. Deblois, C.P. Bean, R.K.A. Wesley, Electrokinetic measurements with submicron particles and pores by the resistive pulse technique, J. Colloid Interface Sci. 61 (1977) 323.

[45] L. Nemec, The effectiveness of iR compensation in controlled-potential polarography, J. Electroanal. Chem. 8 (1964) 166.

[46] P. Vanysek, Two common electroanalytical techniques - Cyclic voltammetry and impedance. Capacitance data from cyclic voltammetry, ECS Trans. 41 (2012) 15.

[47] M.E. Orazem, B. Tribollet, Electrochemical Impedance Spectroscopy, John Wiley and Sons Inc., New Jersey, 2008, pp. 95.

[48] S.E.F. Kleijn, A.I. Yanson, M.T.M. Koper, Electrochemical characterization of nano-sized gold electrodes fabricated by nano-lithography, J. Electroanal. Chem. 666 (2012) 19.

[49] N. Godino, X. Borrise, F.X. Munoz, F.J. del Campo, R.G. Compton, Mass transport to nanoelectrode arrays and limitations of the diffusion domain approach: Theory and experiment, J. Phys. Chem. C 113 (2009) 11119.

[50] D.C. Harris, Quantitative Chemical Analysis, 5th ed., W. H. Freeman and Company, New York, 1998, pp. 483-487.

[51] D. Menshykau, X.J. Huang, N.V. Rees, F.J. del Campo, F.X. Munoz, R.G. Compton, Investigating the concept of diffusional independence. Potential step transients at nano- and micro-electrode arrays: Theory and experiment, Analyst 134 (2009) 343.

[52] Y. Saito, A theoretical study on the diffusion current at the stationary electrodes of circular and narrow band types, Rev. Polarog. 15 (1968) 177.

[53] J. Heinze, Ultramicroelectrodes in electrochemistry, Angew. Chem. Int. Ed. Engl. 32 (1993) 1268.

[54] K.B. Oldham, All steady-state microelectrodes have the same "iR drop", J. Electroanal. Chem. 237 (1987) 303.

[55] S. Bruckenstein, Ohmic potential drop at electrodes exhibiting steady-state diffusional currents, Anal. Chem. 59 (1987) 2098. 
Table 1. Geometric, electrical and temporal behaviour of the three nanolTIES arrays studied in this work by potential step chronoamperometry, and comparison with a single microITIES.

\begin{tabular}{|c|c|c|c|c|}
\hline & \multicolumn{3}{|c|}{ NanolTIES Arrays } & \multirow{2}{*}{$\begin{array}{l}\begin{array}{l}\text { Single } \\
\text { microlTIES [18] }\end{array} \\
5000\end{array}$} \\
\hline Pore radius (nm) & 75 & 50 & 17 & \\
\hline No. of pores $N_{p}$ & 400 & 400 & 400 & 1 \\
\hline $\begin{array}{l}\text { Total ITIES cross-sectional } \\
\text { area }\left(\mathrm{m}^{2}\right)\end{array}$ & $7.07 \times 10^{-12}$ & $3.14 \times 10^{-12}$ & $0.36 \times 10^{-12}$ & $78.5 \times 10^{-12}$ \\
\hline $\begin{array}{l}\text { Experimental capacitance, } \\
C_{\text {expt }}(\mathrm{F})^{\wedge}\end{array}$ & $4.83 \times 10^{-9}$ & $4.44 \times 10^{-9}$ & $1.57 \times 10^{-9}$ & $8.00 \times 10^{-12}[33]$ \\
\hline $\begin{array}{l}\text { Uncompensated resistance, } \\
R_{u}(\mathrm{M} \Omega)^{\#}\end{array}$ & 3.42 & 7.30 & 58.57 & 10.00 \\
\hline Cell time constant, $R_{u} C_{\text {expt }}(\mathrm{s})$ & 0.017 & 0.032 & 0.092 & 0.00008 \\
\hline Charging time, $5 R_{u} C_{\text {expt }}(\mathrm{s})$ & 0.08 & 0.16 & 0.46 & 0.0004 \\
\hline $\begin{array}{l}\text { Experimental specific } \\
\text { capacitance, } C_{\text {expt }}^{0}\left(\mathrm{~F} / \mathrm{m}^{2}\right)\end{array}$ & 630 & 1450 & 4320 & - \\
\hline Response time (s) & $6 \pm 1$ & $6 \pm 1$ & $6 \pm 1$ & - \\
\hline
\end{tabular}

^: Estimated from CV data. \#: total pore and bulk resistance for each membrane calculated using equations 5 and 6. 

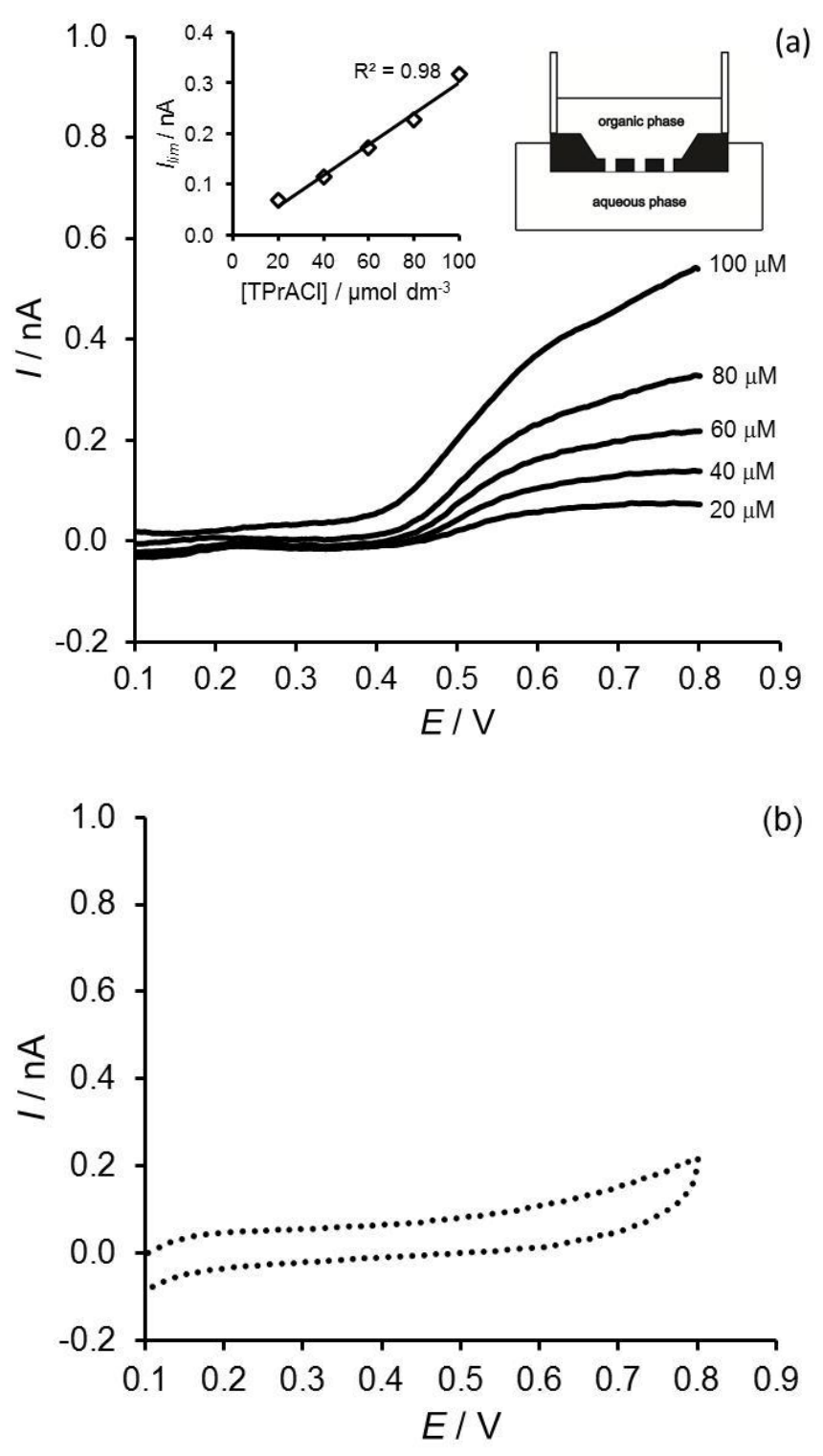

Figure 1. (a) Background-subtracted voltammogram (forward scan) of 20 to $100 \mu \mathrm{M}$ TPrACl at a water | DCH nanoITIES array. The membrane employed comprised 400 pores of $50 \mathrm{~nm}$ pore radius. The foot of the ion transfer wave was at ca. $0.4 \mathrm{~V}$ and the experimental limiting current was determined at approximately $0.6 \mathrm{~V}(200 \mathrm{mV}$ positive of the wave foot) for all concentrations studied. Inset: Calibration graph correlating limiting current and analyte concentration and a diagram (not to scale) showing the cross section of the nanopore membrane filled with the organic phase and in contact with the aqueous phase. (b) Blank $\mathrm{CV}$ at the $50 \mathrm{~nm}$ pore radius 
nanoITIES array. The charging current was determined at $0.2 \mathrm{~V}$, and used with equation 7 to determine the experimental capacitance at $10 \mathrm{mV} \mathrm{s}^{-1}$ sweep rate. 

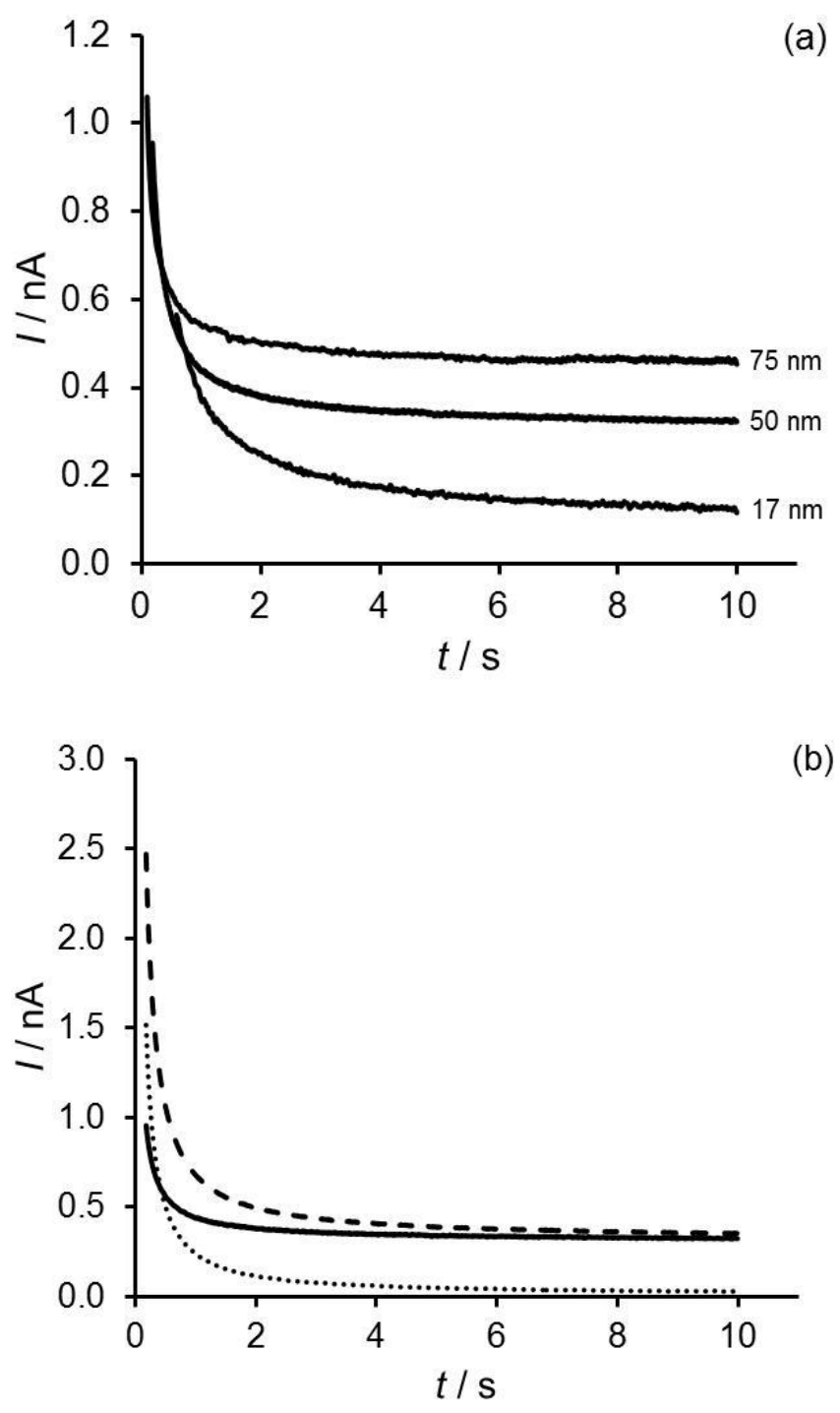

Figure 2. (a) Background-subtracted chronoamperograms of $100 \mu \mathrm{M}$ TPrACl obtained using nanoITIES arrays formed by nanopore array membranes with three different sizes of nanopores (radii of 75, 50 and $17 \mathrm{~nm}$ ). (b) Chronoamperograms at the $50 \mathrm{~nm}$ pore radius nanoITIES array of blank, analyte $(100 \mu \mathrm{M}$ TPrACl) and background-subtracted chronoamperograms, represented by dotted, dashed and solid lines, respectively. 

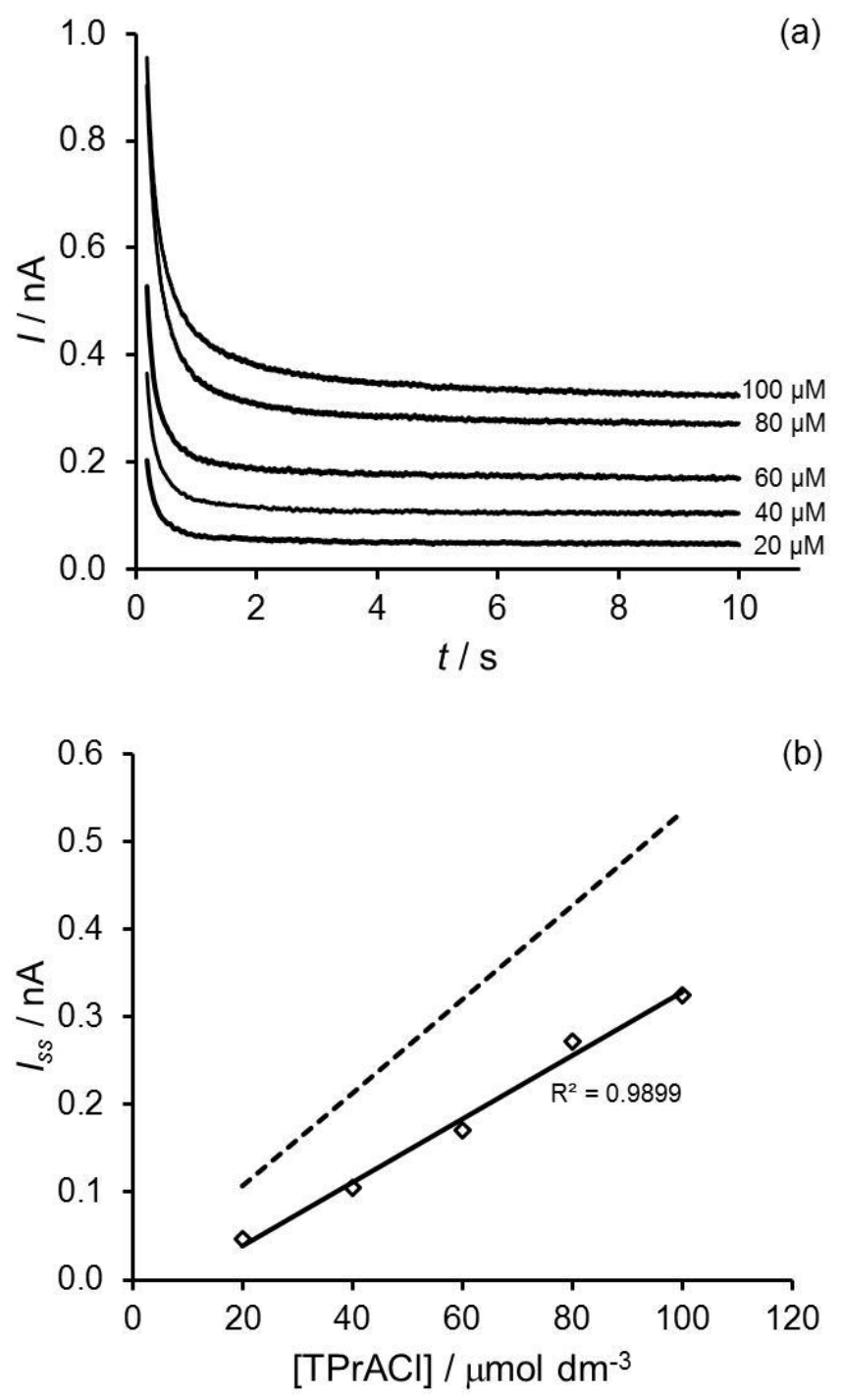

Figure 3. (a) Background-subtracted chronoamperograms of 20 to $100 \mu \mathrm{M}$ TPrACl at a nanoITIES array based on a $50 \mathrm{~nm}$ pore radius membrane. (b) The corresponding calibration curve of the steady-state currents versus the $\operatorname{TPrA}^{+}$ concentration. The dashed line represents the theoretical current calculated using equation (10) while the solid line is the best linear fit to the experimental data. 


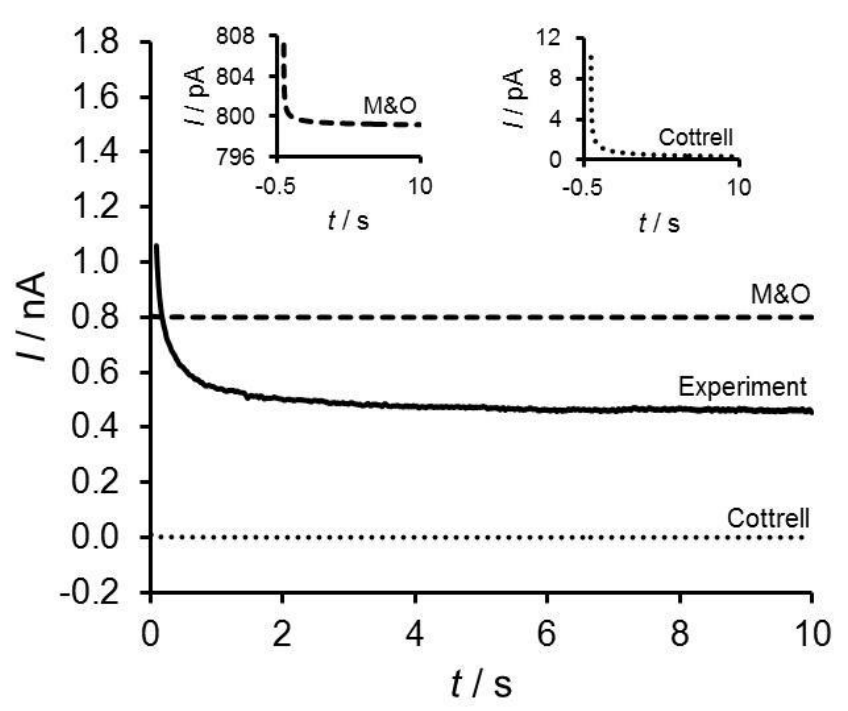

Figure 4. The experimental, Cottrellian and Mahon \& Oldham (M\&O) current transients for $100 \mu \mathrm{M}$ TPrACl at a nanolTIES array formed by $75 \mathrm{~nm}$ pore radius membrane. The inset shows a magnified view of the Cottrellian and Mahon \& Oldham current response. 


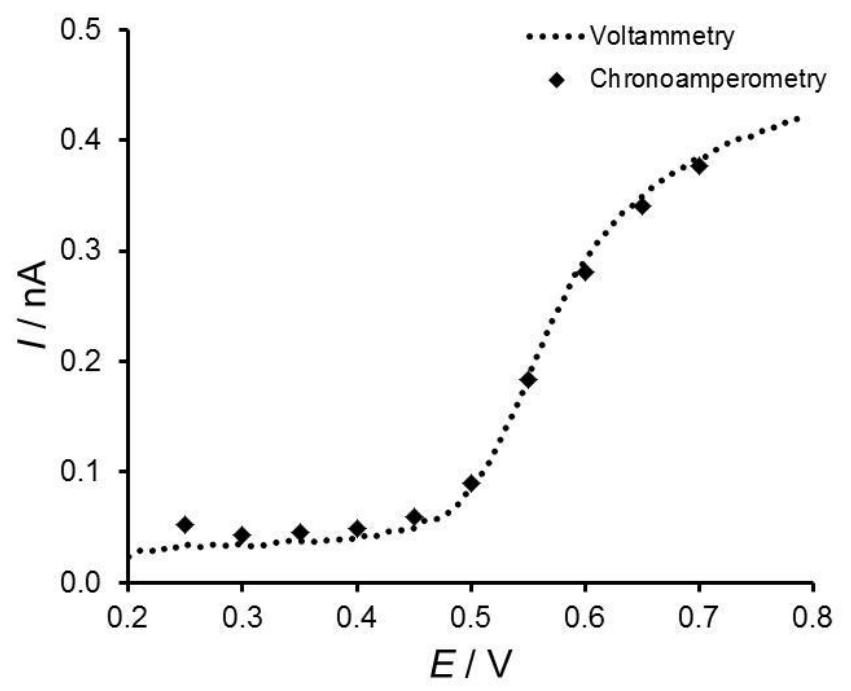

Figure 5. Comparison of the chronoamperometric steady-state current with the voltammetric response at a nanoITIES array formed at a $75 \mathrm{~nm}$ pore radius membrane. Voltammetric sweep rate: $10 \mathrm{mV} \mathrm{s}^{-1}$. 